\title{
The genetic/metabolic transformation concept of carcinogenesis
}

\author{
Leslie C. Costello and Renty B. Franklin \\ Department of Oncology and Diagnostic Sciences, Dental School, and The Greenebaum Cancer \\ Center, University of Maryland, 650 West Baltimore Street, Baltimore, MD 21201, USA \\ Leslie C. Costello: Icostello@umaryland.edu
}

\begin{abstract}
The carcinogenesis process is poorly understood and subject to varying concepts and views. A rejuvenated interest has arisen regarding the role of altered cellular intermediary metabolism in the development and progression of cancer. As a result, differing views of the implications of altered metabolism in the development of cancer exist. None of the concepts recognize and incorporate the principles of cell metabolism to cell activity, which are applicable to all cells including the carcinogenesis process. This presentation incorporates a novel concept of carcinogenesis that includes a "genetic/metabolic" transformation that encompasses these principles of cell metabolism to cell activity. The intermediary metabolism transformation is essential to provide the bioenergetic/ synthetic, growth/proliferation, and migration/invasive events of malignancy. The concept invokes an "oncogenetic transformation" for the development of neoplastic cells from their precursor normal cells; and a required "genetic/metabolic" transformation for facilitation of the development of the neoplastic cells to malignant cells with the manifestation of the malignant process. Such a concept reveals stages and events of carcinogenesis that provide approaches for the identification of biomarkers and for development of therapeutic agents. The presentation discusses the contemporary application of genetics and proteomics to altered cellular metabolism in cancer; and underscores the importance of proper integration of genetics and proteomics with biochemical and metabolic studies, and the consequences of inappropriate studies.
\end{abstract}

\section{Keywords}

Carcinogenesis; Genetic/metabolic transformation; Tumor metabolism; Metabolic malignant transformation

\section{Introduction}

Carcinogenesis is the transformation of normal cells to malignant cells. Despite decades of extensive studies and concepts, the process involved in this transformation remains largely unknown and speculative, as witnessed by differing existing concepts. The dominant contemporary view is reflected in Vogelstein and Kinzler's review [1] that "cancer is, in essence, a genetic disease." This view incorporates the concept that the initiation of cancer is

(C) Springer Science + Business Media, LLC 2011

Correspondence to: Leslie C. Costello, lcostello@umaryland.edu. 
not the result of a single gene mutation or alteration; but requires a series of gene alterations. This view further incorporates the concept of the involvement of (1) mutations that activate oncogenes; (2) mutations that inactivate tumor suppressor genes; and (3) inactivation of stability genes, which then increases mutations of other genes. For our discussion and concept, we will describe these collective genetic events as "oncogenetic transformation," which initiates and results in the genetic transformation of the normal cell to a neoplastic cell, not the transformation of a normal cell to a malignant cell (as will be discussed below). Otherwise, we have no issue with this view of the initiation of the carcinogenesis process. However, the rigidity and interpretation of this concept excludes or lacks the important consideration and incorporation of the principles of the relationship between cellular activity and cellular metabolism, especially intermediary metabolism, as required events in the carcinogenesis process. This is the focus of our discussion and concept.

\section{Axioms for the relationship of cell metabolism to cell activity: application in carcinogenesis}

Any concept of carcinogenesis must incorporate an accurate understanding of fundamental cellular relationships. One such indispensable relationship is the interdependence of a cell's activity and its metabolism [2-5]. The relationship is defined by the following principles that we refer to as "axioms of cell activity and cell metabolism."

1. The existing intermediary metabolism of a cell provides the bioenergetic/synthetic/ catabolic requirements that are essential for the manifestation of the cell's current activity (e.g., function, growth, proliferation, differentiation).

2. When the activity of a cell changes, its metabolism must also be altered to provide new bioenergetic/synthetic/ catabolic requirements for the cell's changing activity.

3. Malignant cells are derived from normal cells that have undergone genetic transformation to neoplastic cells that have malignant potential.

4. Manifestation of the malignant potential of the neoplastic cell necessitates alterations in its metabolism (i.e., metabolic transformation) to provide the bioenergetic, synthetic, and catabolic requirements of malignancy.

5. In the absence of the metabolic transformation, the neoplastic cell will not progress to a malignant cell with its manifestation of malignancy. Conversely, the metabolic transformation, in the absence of the genetic transformation to a neoplastic cell, will not cause malignancy.

We submit that axioms 1 and 2 are irrefutable universal principles applicable to all cells. "Metabolism" is one of the attributes of life, and these axioms describe its relationship to cell activity. Axioms 3-5 are applications of axioms 1 and 2 to cancer, and, in our view, must be involved and applied as essential requirements in the transformation of normal cells to malignant cells and the process of malignancy. Also, we will present another axiom (axiom 6) later in this presentation. 


\section{The activity of a cell dictates its metabolism: malignant cells are "auto- parasitic" cells}

The preceding discussion dictates that an understanding of a cell's activity will provide information concerning the required metabolic state of the cell. So, defining the activity of the malignant cell relative to its precursor normal cell will provide information regarding required metabolic changes that must accompany this transformation. We describe malignant cells as "auto-parasitic" cells [2-5]. As such, malignant cells have one purpose in life, i.e., to grow and to proliferate. It lives to reproduce, to ensure its generational propagation. It does so at the expense and destruction of its host. These are the criteria of a parasitic lifestyle. The difference between malignant cells and "true" parasites is that the latter arises external to the host, invades the host, and has life cycle stages that leave the host in search of a new host. Malignant cells arise from the host's cells, destroy the host, and ultimately themselves; thereby the term "auto-parasitic" cells. It is interesting to note the similar view that was expressed by Baggetto [6 “...cancer cells will kill neighbouring cells... since it is compatible with the existence of these parasites of the living organisms." The malignant cells exhibit two essential activities for their progression and propagation: (1) growth and proliferation, (2) invasion and migration. The latter are life-cycle activities in support of the former.

With this understanding, it is confusing, in our view, to consider malignant cells as "well differentiated," "moderately differentiated," or "undifferentiated" cells. This terminology might be appropriate for the histopathological characterization for grading of malignancy from early stage to advancing stages; at the same time, however, it misrepresents the transformational development of malignancy. In so doing, it characterizes malignant cells in the same category as normal undifferentiated cells (e.g., stem cells, mesenchymal cells and others that we will refer to collectively as "stem" cells). Stem cells, like malignant cells, also exist to grow and proliferate; but they do so for the purpose of differentiating into specialized cells that perform specific functions. They proliferate to maintain a continual population for further differentiation [2-4, 7]. However, unlike the parasitic malignant cells, these cells grow/proliferate in a regulated manner in harmony with the host tissue, i.e. they exhibit a symbiotic lifestyle. In this sense, these are "sane" cells and malignant cells are "insane" cells. Therefore, the histopathological grading characteristic of undifferentiated malignancy implies a reversion of the malignant cell toward its earlier origin of the sane cell from its "undifferentiated stem cell origin." The malignant cell never reverts to a nonmalignant dedifferentiated sane cell activity. However, this histopathological characterization is appropriate for its purpose; but the context relative to the activity/ functional relationship of transformation of normal cells to malignant cells must be separated from the histopathological context.

The intermediary metabolism of the malignant cells must provide the bioenergetic, synthetic and catabolic requirements for these activities. For example, the malignant cell activity of growth and proliferation dictates that the malignant cells become de novo lipogenic/ cholesterogenic cells $[2,4,5]$. In most cases, the precursor sane cells that become neoplastic cells are not lipogenic/cholesterogenic proliferating cells. Therefore, the proliferating 
malignant cells must undergo a metabolic transformation directed at de novo lipogenesis/ cholesterogenesis, which is essential to meet the membraneogenesis and other requirements of proliferation. Bioenergetic requirements to support growth/proliferation as well as motility/invasion must also be achieved. This is an example of the required metabolic adaptations in support of the manifestation of the malignant activities of the neoplastic malignant cells. In the absence of these metabolic activities, the neoplastic cells will not progress to malignancy.

\section{In situ environment of the malignant cell impacts its metabolism}

Regardless of the metabolic requirements that are essential to their malignant activities, the malignant cells must achieve these metabolic requirements through their interaction with the host's tissue environment. The malignant cells are dependent on the host tissue and circulation for their supply of oxygen and nutrients, and for the elimination/ removal of the potentially toxic by-products of their metabolism. In particular, for solid tumors, the malignant cells are subjected to a changing environment as they grow and progress. Most notable is the influence of the availability of oxygen and micronutrients derived from circulation. The initiation of the malignant cell activity is followed by growth and proliferation that results in an increasing mass of malignant cells. This exposes the population of malignant cells to different gradients of oxygen ranging from normoxia through hypoxia toward anoxia. Thus, within the total population of malignant cells, subpopulations with differing metabolic activities will exist. Therefore a tumor mass cannot be expected to consist of a uniform population of malignant cells with the same metabolic profile $[2,4,5,8]$.

As the population of proliferating and growing cells increases, and the tumor mass increases, the perfusion of the cells by the host circulation/interstitial fluid decreases. This leads to hypoxia/anoxia, limited availability of glucose and other nutrients, and limited removal of $\mathrm{CO}_{2}$ and products of metabolism [2,8]. Under these conditions of insufficient host perfusion fluid, malignant cell proliferation and other activities will become quiescent; and the malignant cell will direct its genetic/metabolic activity to facilitation of angiogenesis and restoration of an effective perfusion condition to support proliferation/growth and migration/ invasion requirements. In other words, a refurbished perfusate is an essential environmental condition for tumor progression. Indeed, the successful evolution of malignant cells has resulted from adaptive capabilities to confront and to overcome this adversity. For example, they up-regulate hypoxia-inducible factors and stimulate angiogenesis to create the circulation and environment that allows their further progression [9-11].

Therefore, the cycle of malignancy involves periods of growth and proliferation and periods of arrest to "refuel" the environment; all of which accommodate the metabolic requirements of the malignant cells. To optimize their parasitic existence, some malignant cells will vacate the primary site of their development and metastasize to other host tissue sites to invade and to continue their parasitic existence. This involves migration/invasive activities, which require different metabolic relationships than proliferation/growth. Thus, the capability and stages of metastasis are invoked. The vascularization and distant tissue site invasion of the malignant cells provide different environmental conditions that likely affect 
the metabolism of the metastatic cells. Very little is known concerning the in situ metabolic relationships of these cells.

The understanding of all of these relationships dictates that any studies of tumor cell metabolism must be extrapolated and related to the realities of the in situ environment of the malignant cells. One should recognize that in vitro studies with tumor cells do not represent the in situ conditions of malignant cells. The idea that tumor cells by and large exhibit a high aerobic glycolysis and impaired mitochondrial respiration is much too rigid for an understanding of carcinogenesis; especially for the importance of metabolic transformations.

\section{Views on the role of tumor cell metabolism in carcinogenesis}

There is little question that the metabolism of malignant cells is different from that of their precursor normal cells. Having acknowledged this, we hasten to add that the view that all tumor cells exhibit the same altered metabolic pathways is untenable. This view has arisen from the hallmark tumor metabolism studies of Warburg and colleagues [12]. Arising from those studies is the concept of impaired mitochondrial respiration and high aerobic glycolysis as being common and characteristic to all tumor cells. The rigidity of the application of this relationship in carcinogenesis is problematic for several reasons as described in the preceding section. This was emphasized by Moreadith and Lehninger [13] in their statement that "It has been further concluded that glutamine, not glucose, is the major energy source for HeLa cells even in the presence of physiological levels of glucose.... Such studies have therefore cast some doubt on the essentiality of the conversion of glucose to lactate by aerobic glycolysis for tumor cells."

Warburg [14] presented the concept that the permanent impaired mitochondrial respiration of normal cells results in the survival of "strongly fermenting" cells as a successful adaptation to the mitochondrial dysfunction, and these cells are the cancer cells, i.e., a natural selection process. However, at that time, this concept of Warburg's was strongly and passionately argued between proponents and detractors (e.g., [15]). One must recognize that this was a time that preceded the era of knowledge of molecular genetics and genomics; so the exclusion of the requirement for the genetic involvement in the transformation of normal to malignant cells is understandable. However, one cannot dismiss the possibility and perhaps likelihood that conditions associated with impaired mitochondrial metabolism and function (such as production of high levels of ROS) could initiate or lead to "oncogenetic" changes that initiate carcinogenesis.

Another view is exemplified by Seyfried and Shelton [16] that cancer is a metabolic disease. This view, similar to and supportive of Warburg's concept, describes impaired mitochondrial metabolism of normal cells as the cause of the development of malignant cells; and genetic changes are secondarily induced by the impaired metabolism. The authors state "The gene theory of cancer would argue that mitochondrial dysfunction is an effect rather than a cause of cancer, whereas the metabolic impairment theory would argue the reverse"; and they propose the latter to be the more appropriate concept.

The views as represented above are consistent in the contention that impaired metabolism is the causative factor of all cancers. Warburg was very careful to emphasize the importance of 
"irreversible permanent" mitochondrial respiratory damage as opposed to "reversible" malfunction in his concept. Nevertheless, descriptions such as "impaired cellular energy metabolism"; "aberrant metabolism," "metabolic impairment"; "tumors do not express normal respiration"; all lead to the presumption that the altered metabolism in the malignant cell from that of the normal cell represents a dysfunctional or abnormal metabolism in the malignant cell. This view eliminates any consideration of the more likely, in our opinion, condition that this altered metabolism can be the "normal and required metabolism" of the malignant cell. Then, this is an evolutionary metabolic adaptation required for malignant cells to be malignant cells that manifest the activities of malignancy, i.e., the application of axioms $1,2,4$, and 5 .

A somewhat different view is expressed by Hanahan and Weinberg [10] which they describe as "reprogramming of energy metabolism." They recognized and added this relevance of energy metabolism as an important factor in the development cancer, which was not included in their earlier concept [9]. They present the view that “...major reprogramming of cellular energy metabolism in order to support continuous cell growth and proliferation, replacing the metabolic program that operates in most normal tissues and fuels the physiological operations of the associated cells...." This is akin to our earlier descriptions of the required role of altered metabolism in the development of malignancy [2-5]. However, we question their characterization of "deregulating" cellular energy as a core hallmark capability of cancer cells," since "deregulation" can imply an aberrant or impaired metabolism.

Consider the functional metabolic relationship in prostate. The Noble laureate, Dr. Huggins, stated [17 "From the standpoint of oxidative metabolism the prostate gland belongs with tissues that possessing a low oxygen consumption ... and a high anaerobic glycolysis and a considerable aerobic glycolysis." Additionally, normal prostate mitochondria exhibit State 3 and State 4 respiration that is $80 \%$ lower than that of liver mitochondria; and exhibit 50$75 \%$ lower activity of the electron transport components [18]. In addition, these normal prostate cells have a truncated Krebs cycle with minimal oxidation of citrate [19]. This is a metabolic profile that mimics the tumor cell metabolic profile more so than the metabolic profile of many other normal mammalian cells. For the normal prostate cells, these metabolic conditions cannot be characterized as "impaired cellular energy metabolism" or "aberrant metabolism." This is the normal metabolic relationship that is required for the normal functional activity of these cells (i.e., the application of axiom 1). It is also obvious that these normal epithelial cells do not exist as malignant cells as a result of this metabolic profile. However, if this metabolic profile transformation occurred in liver cells, it would likely represents an "impaired cellular metabolism" with consequences to the liver cells. Would this directly transform the normal liver cell to a malignant cell? Likely not, if we apply axiom 5 "... Conversely, the metabolic transformation, in the absence of the genetic transformation to a neoplastic cell, will not cause malignancy."

Moreover, as discussed above, the malignant cells will have changing metabolism requirements as they progress. The same bioenergetic and biosynthetic requirements for metabolic activities and pathways apply to normal and malignant cells. The differences are which metabolic activities become prominent relative to the activity of the cell at that time. 
The axioms of cell activity and cell function apply to all cases. How does one explain that a permanent irreversible damaged mitochondrial metabolism and its consequences can exist in malignant cells, when these cells have changing metabolic requirements as malignancy develops and progresses, which require efficient mitochondrial activities?

We reiterate that dysfunctional metabolism and particularly dysfunctional mitochondrial metabolism can induce gene expression changes, which then can lead to the transformation of the normal cell to a neoplastic cell. (Note that we employ the terminology "neoplastic" cell and not "malignant" cell; which is an important distinction that will be described below.) This neoplastic transformation will not occur in the absence of genetic involvement. The important point is that altered metabolism is not necessarily impaired or dysfunctional metabolism; and a distinction must be made between the two. Moreover, the widespread view that all cells rely on the same metabolic profile, and all tumor cells exhibit the same "dysfunctional" metabolic profile, is untenable.

\section{The concept of the "genetic/metabolic" transformation in the carcinogenesis process}

The issue at hand is not "Does the malignant cell exhibit changes in metabolism from that of its normal cell?" Nor is it "What are the specific changes in metabolism?" The issue is "What is the role and implication of the changes in metabolism in the process of carcinogenesis?" The essential point is that most views do not encompass a concept that altered metabolism in the transformation of normal cells to malignant cells is an event that is required for the development of the malignant cell and the manifestation of malignancy. The consideration that is lacking in the other concepts is that the malignant process (proliferation/growth; migration/invasion) requires altered metabolism to provide the energetic/synthetic/catabolic requirements for these activities. As such, the principles of cell activity and cell metabolism relationships must be incorporated in the concept of carcinogenesis.

In large measure, the divergent views regarding the role and implication of altered metabolism in carcinogenesis arise from one's perception or understanding of the events in carcinogenesis. Many, and possibly most, concepts of the carcinogenesis process do not include or acknowledge any important role or involvement of altered metabolism; particularly cellular intermediary metabolism. Such concepts of the events involved and required for the progressive transformation of normal cells to malignant cells completely ignore the principles of the relationship of cell metabolism to cell activity, i.e., adherence to the axioms described above. Because of the absence and exclusion of this understanding, the various concepts of the carcinogenesis process do not reflect the important requirement for a "genetic/metabolic" transformation event in the development of malignancy. This, in our view, results in a deficient understanding of the development and progression of cancers. As a result, serious consequences arise in regard to elucidating the factors and events in the etiology and progression of malignancy; in regard to the identification of important biomarkers of early malignancy and even premalignant at-risk subjects; and in regard to the development of efficacious cancer therapeutic and possibly preventive agents. 
In consideration of the background presented above, we present our concept of the role and implication of altered cell metabolism in the carcinogenesis process (Fig. 1). We begin with the incorporation of the concept [1] that "cancer is, in essence, a genetic disease." However, our concept imposes some modification from the general application of this genetic concept. As we defined in the "Introduction" section, the initiating genetic changes will collectively be described as the "oncogenetic transformation." Here, we make a critical distinction that is not included or reflected in most, perhaps all, other concepts of carcinogenesis. We reject the view that the "oncogenetic transformation" results in the transformation of a normal cell to a "malignant" cell. We present the view that the "oncogenetic transformation" transforms the normal cell to a "neoplastic" cell, i.e., a genetically transformed cell that has potential malignant capability. This constitutes the "neoplastic transformation phase" of carcinogenesis.

When viewed with this perspective, a critical point surfaces. "What is required to transform the "neoplastic" cell to a "malignant cell" with full capability to manifest the required events of the malignant process. This requires "genetic/metabolic" events downstream from the neoplastic cell, which occur via oncogenetic-initiated cascading and progressing signaling pathways and factors. We refer to these events as the "facilitative phase" required for the transformation of the neoplastic cell to the malignant cell with the capability of malignancy. In so doing, the concept incorporates the involvement of "premalignant" cells as transitional cells in the progression of the facilitating events and changes leading to the malignant cells. This also raises the issue that the term "preneoplastic" should not be used as a synonym for "premalignant" since they are different stages in the carcinogenesis process.

Critical to the facilitative phase is the metabolic transformation, because axioms 1 and 2 are indispensable for all cells; and axioms 3-5 specifically apply axioms 1 and 2 to cancer. This metabolic transformation provides the bioenergetic/synthetic/catabolic requirements for the process of malignancy (growth/proliferation and migration/invasion); which then becomes the "malignant phase" of carcinogenesis. In this transformation another distinction must be made. The facilitative events will also involve genetic changes in association with the metabolic events; which are separate from the genes that comprise the oncogenetic transformation and initiation of the neoplastic phase. This is illustrated in Fig. 1 relative to the carcinogenesis of prostate cancer.

\section{Prostate cancer as a model for the genetic/metabolic carcinogenesis concept}

Our genetic/metabolic concept arose and evolved from our studies over the past 20 years regarding zinc and citrate-associated metabolic relationships in normal prostate and prostate cancer (Fig. 1b). It is now established that zinc exhibits tumor suppressor actions on malignant prostate cells, which include altered metabolism, inhibition of growth/ proliferation, and inhibition of migration/invasion [3, 20]. Normal prostate secretory epithelial cells contain high zinc levels that inhibit $\mathrm{m}$-aconitase activity and citrate oxidation to allow for their function to secrete high levels of citrate in prostatic fluid [3, 19]. In prostate cancer, the malignant cells have lost the ability to accumulate zinc, and citrate is oxidized via the Krebs cycle rather than accumulating for secretion. ZIP1 is the functional 
zinc uptake transporter associated with the high accumulation of zinc in the normal cells. In the malignant cells, ZIP1 is down-regulated, which prevents the uptake and accumulation of zinc. The silencing of ZIP1 and the decrease in zinc constitute a genetic/metabolic transformation that removes the tumor suppressor effects of zinc and facilitates the development of the neoplastic cell to the malignant cell with the manifestation of malignancy.

Consistent with this concept is the evidence that this genetic/metabolic transformation occurs in early malignancy and in premalignant cells/lesions [21-24]. In their prostate cancer in situ zinc studies, Cortesi et al. [22] noted that “...the zinc depletion occurs not only in the cancerous tissue segments but also, though less pronouncedly, in the Non-Cancer components surrounding the lesion.... This observation is consistent with the conclusions of Costello et al. that the zinc depletion is an early step in the cancer proliferation process and that zinc depletion precedes the transformation of cells from normal to cancerous type. It is well possible that although PCa has not been observed by the pathologist in these regions, the cellular precursor for its appearance is already present...." Corroborating this view, Johnson et al. [23] state "...we demonstrate that $\mathrm{Zn}$ is depleted from the neoplastic as well as pre-neoplastic prostatic glandular epithelial cells." Relative to citrate changes, which are due to zinc changes, Cooper and Farid [24] noted that altered citrate metabolism "occurs early in malignancy, which precedes the histopathological identification of malignant prostate cells." Thus, strong supporting evidence exists for existence of this genetic/ metabolic transformation as an important event in the carcinogenesis process for prostate cancer as shown in Fig. 1a. Although the specific genetic/metabolic transformations will vary with the metabolic profiles and requirements associated with each specific cancer, genetic/metabolic transformations apply to all cancers.

\section{Axiom 6. Genetic transformations and proteomic changes have little relevancy if the genetic/proteomic alterations are not manifested as changes in cell metabolism and function. The absence of identified genetic transformations and proteomic changes does not demonstrate the absence of changes in cell metabolism and function}

The prostate cancer model also reveals some important considerations and issues. The contemporary marriage of genomics and proteomics with the resurgent interest in altered cellular metabolism in cancer and other pathologies has opened the door to new advancements and understanding of factors and mechanisms, which heretofore was not achievable. Unfortunately, this has also been accompanied by misinformation and inappropriate translational interpretations resulting from implications of genetic and proteomic studies of cellular metabolism in relation to cancer and other diseases. This caused us to raise and to describe this issue in earlier reports [4, 5, 25], which gave rise to axiom 6 "Genetic transformations and proteomic changes have little relevancy if the genetic/ proteomic alterations are not manifested as changes in cell metabolism and function." However, this description has proven to be insufficient in dealing with the issues and problems that have arisen from reports involving genetic and proteomic studies of cell 
metabolism. Therefore, we have revised axiom 6 as follows: "Genetic transformations and proteomic changes have little relevancy if the genetic/proteomic alterations are not manifested as changes in cell metabolism and function. The absence of identified genetic transformations and proteomic changes does not demonstrate the absence of changes in cell metabolism and function." This is an essential axiom when viewed in the context of the important role of the genetic/metabolic transformation in the process of carcinogenesis.

It is necessary for one to have knowledge and understanding of important fundamental relationships and principles of cell metabolism, biochemistry, enzymology and enzyme kinetics (that apply to enzymes and transporters), which are essential to the application and interpretation of genetic and proteomic studies and their results $[4,5,25,26]$. It is not possible to review and describe this background in this report, much of which is already available $[2,4,5,25]$. Instead, the following will provide some representative considerations that are evident from the prostate cancer model.

The key metabolic transformation in prostate cancer is the increased citrate oxidation that occurs in the malignant cells. This is due to increased m-aconitase activity as compared to low activity and low citrate oxidation in the normal epithelial cells. Immunohistochemistry for maconitase in normal prostate and prostate cancer tissues reveals no difference in the enzyme abundance [27]. Based on this proteomic analysis, the potential conclusion would be that $\mathrm{m}$-aconitase is not involved in this metabolic transformation. However, the low activity of m-aconitase in situ is due to its inhibition by high levels of zinc in normal prostate epithelium; which does not exist in the malignant cells that possess low levels of zinc. This revelation could only be identified by metabolic studies. This is corroborated by the measurements of citrate and isocitrate (m-aconitase substrate and product) levels in the prostate tissues, which changes from a 30:1 ratio in normal tissue to 10:1 in malignant tissue $[19,28]$.

The important gene in this metabolic transformation is ZIP1, which is the functional zinc uptake transporter in normal prostate epithelial cells; and which is down-regulated in the malignant cells in situ [3]. ZIP2 and ZIP3 are also down-regulated in prostate cancer. However, immunohistochemistry of tissue sections reveals that ZIP1 is localized at the basolateral membrane of the glandular epithelium; whereas ZIP2 and ZIP3 are localized at the apical membrane. Thus ZIP1 is the functional transporter for the uptake and accumulation of zinc from circulation. Gene microarrays and Western blots would not identify this important cell function relationship, and would result in misleading interpretations and conclusions.

The example discussed above raises another issue. Despite the fact that ZIP1is silenced in situ in prostate malignancy, the malignant cell lines such as PC-3 and LNCaP exhibit constitutive expression of ZIP1 transporter. This is likely due to the removal of epigenetic silencing conditions that exist in situ. Thus, identification of the in situ status and importance of altered ZIP1 expression in prostate cancer is not revealed by cell lines, and the latter leads to misrepresentation of the important genetic/metabolic events in prostate carcinogenesis. 
Another issue resides in the application of quantitative relevancy of genetic expression and gene product abundance changes to cellular metabolism and activity. All gene products are considered to be of similar consequence, which leads to interpretations that the magnitude of a change is proportional to the influence and relevance on the cells activity. This is especially a misrepresentation when applied to cellular regulatory enzymes of intermediary metabolism and transporters, which we define as "metabolic genes." Metabolic gene products exist in low abundance compared to the high abundance of proteins such as structural/skeletal proteins and secretory/digestive enzymes. A change of 1- to 2-fold in the regulatory enzyme or transporter will have significant effect in altering the activity in the cell, as is represented by ZIP1 in prostate cancer. In fact it makes little sense for a 10-fold change, if this exceeds the $V_{\max }$ of their cellular activity. Yet such changes are considered to be quantitatively irrelevant; and even considered to be insignificant based on the stringency imposed for gene microarray analyses.

\section{Concluding remarks}

The carcinogenesis process must include the accommodation of the principles of the relationships of cell metabolism to cell activity in the transformation of normal cells to malignant cells. Our concept incorporates the genetic/metabolic required event in the facilitation of the development of the malignant cell from the neoplastic cell and the manifestation of the malignant process. This concept provides a new view of required altered metabolism and associated genetic events in the carcinogenesis process for all cancers. When appropriately applied to cell metabolism and function, genetic and proteomic studies and technology will contribute immensely to the identification and understanding of factors and mechanisms associated with the genetic/metabolic transformation in specific cancers. The inclusion of the genetic/metabolic transformation in the carcinogenesis process reveals new targets for identification of early biomarkers and for development of therapeutic agents for cancers.

\section{Acknowledgments}

This report and the studies of LCC and RBF cited herein were supported in part by NIH grants CA79903, DK076783, and DK42839.

\section{References}

1. Vogelstein B, Kinzler KW. Cancer genes and the pathways they control. Nature Medicine. 2004; 10(8):789-799.

2. Costello LC, Franklin RB. 'Why do tumour cells glycolyse?': from glycolysis through citrate to lipogenesis. Molecular and Cellular Biochemistry. 2005; 280(1-2):1-8. [PubMed: 16511951]

3. Costello LC, Franklin RB. The clinical relevance of the metabolism of prostate cancer; zinc and tumor suppression: connecting the dots. Molecular Cancer. 2006

4. Costello, LC.; Franklin, RB. Integration of genetic, proteomic, and metabolic approaches in tumor cell metabolism. In: Singh, K.; Costello, LC., editors. Mitochondria and cancer . New York: Springer; 2009. p. 79-92.

5. Costello, LC.; Franklin, RB. Transformations of malignant cells (an overview). In: Hayat, MA., editor. Methods of cancer diagnosis, therapy, and prognosis. New York: Springer; 2008. p. 3-16.

6. Baggetto LG. Deviant energetic metabolism of glycolytic cancer cells. Biochimie. 1992; 74(11): 959-974. [PubMed: 1477140] 
7. Kasper S. Stem cells: the root of prostate cancer? Journal of Cell Physiology. 2008; 216(2):332336.

8. Dang CV, Samenza GL. Oncogenic alterations of metabolism. Trends in Biological Sciences. 1999; 24(2):68-72.

9. Hanahan D, Weinberg RA. The hallmarks of cancer. Cell. 2000; 100(1):57-70. [PubMed: 10647931]

10. Hanahan D, Weinberg RA. Hallmarks of cancer: the next generation. Cell. 2011; 144(5):646-674. [PubMed: 21376230]

11. Alqawi O, Wang HP, Espiritu M, Singh G. Chronic hypoxia promotes an aggressive phenotype in rat prostate cancer cells. Free Radical Research. 2007; 41(7):788-797. [PubMed: 17577739]

12. Warburg O, Wind F, Negelein E. The metabolism of tumors in the body. Journal of General Physiology. 1927; 8(6):519-530. [PubMed: 19872213]

13. Moreadith RW, Lehninger AL. The pathways of glutamate and glutamine oxidation by tumor cell mitochondria. Role of mitochondrial NAD $(\mathrm{P})+-$ dependent malic enzyme. Journal of Biological Chemistry. 1984; 59(10):6215-6221. [PubMed: 6144677]

14. Warburg O. On the origin of cancer cells. Science. 1956; 123(3191):309-314. [PubMed: 13298683]

15. Weinhouse S, Warburg O, Burk D, Schade AL. On respiratory impairment in cancer cells. Science. 1956; 123(3191):309-314. [PubMed: 13298683]

16. Seyfried TN, Shelton LM. Cancer as a metabolic disease. Nutrition and Metabolism. 2010

17. Huggins C. The prostate secretion. Harvey Lectures. 1946; 42:148-193.

18. Costello LC, Guan Z, Kukoyi B, Feng P, Franklin RB. Terminal oxidation and the effects of zinc in prostate versus liver mitochondria. Mitochondrion. 2004; 4(4):331-338. [PubMed: 16120396]

19. Costello LC, Franklin RB, Feng P. Mitochondrial function, zinc, and intermediary metabolism relationships in normal prostate and prostate cancer. Mitochondrion. 2005; 5(3):143-153. [PubMed: 16050980]

20. Franklin RB, Costello LC. Zinc as an anti-tumor agent in prostate cancer and in other cancers. Archives of Biochemistry and Biophysics. 2007; 463(2):211-217. [PubMed: 17400177]

21. Franklin RB, Feng P, Milon BC, Desouki MM, Singh KK, Kajdacsy-Balla A, et al. hZIP1 zinc uptake transporter down regulation and zinc depletion in prostate cancer. Molecular Cancer. 2005; 4(32):13. [PubMed: 15811177]

22. Cortesi M, Fridman E, Volkov A, Shilstein SS, Chechik R, Breskin A, Vartsky D, Kleinman N, Kogan G, Moriel E, Gladysh V, Huszar M, Ramon J, Raviv G, et al. Clinical assessment of the cancer diagnostic value of prostatic zinc: a comprehensive needle-biopsy study. Prostate. 2008; 68(9):994-1006. [PubMed: 18386292]

23. Johnson LA, Kanak MA, Kajdacsy-Balla A, Pestaner JP, Bagasra O. Differential zinc accumulation and expression of human zinc transporter 1 (hZIP1) in prostate glands. Methods. 2010; 52(4):316-321. [PubMed: 20705137]

24. Cooper JE, Farid I. The role of citric acid in the physiology of the prostate: lactic/citrate ratios in benign and malignant prostatic homogenates as an index of prostatic malignancy. Journal of Urology. 1964; 92:533-536. [PubMed: 14226486]

25. Costello LC, Franklin RB. Tumor cell metabolism: the marriage of molecular genetics and proteomics with cellular intermediary metabolism; proceed with caution! Molecular Cancer. doi. 2006

26. Costello LC. The effect of contemporary education and training of biomedical scientists on present and future medical research. Academic Medicine. 2009; 84(4):459-463. [PubMed: 19318778]

27. Singh KK, Desouki MM, Franklin RB, Costello LC. Mitochondrial aconitase and citrate metabolism in malignant and nonmalignant human prostate tissues. Molecular Cancer.

28. Costello, LC.; Littleton, GK.; Franklin, RB. Regulation of citrate-related metabolism in normal and neoplastic prostate. In: Sharma, RK.; Criss, WE., editors. Endocrine control in neoplasia. New York: Raven; 1978. p. 303-314. 

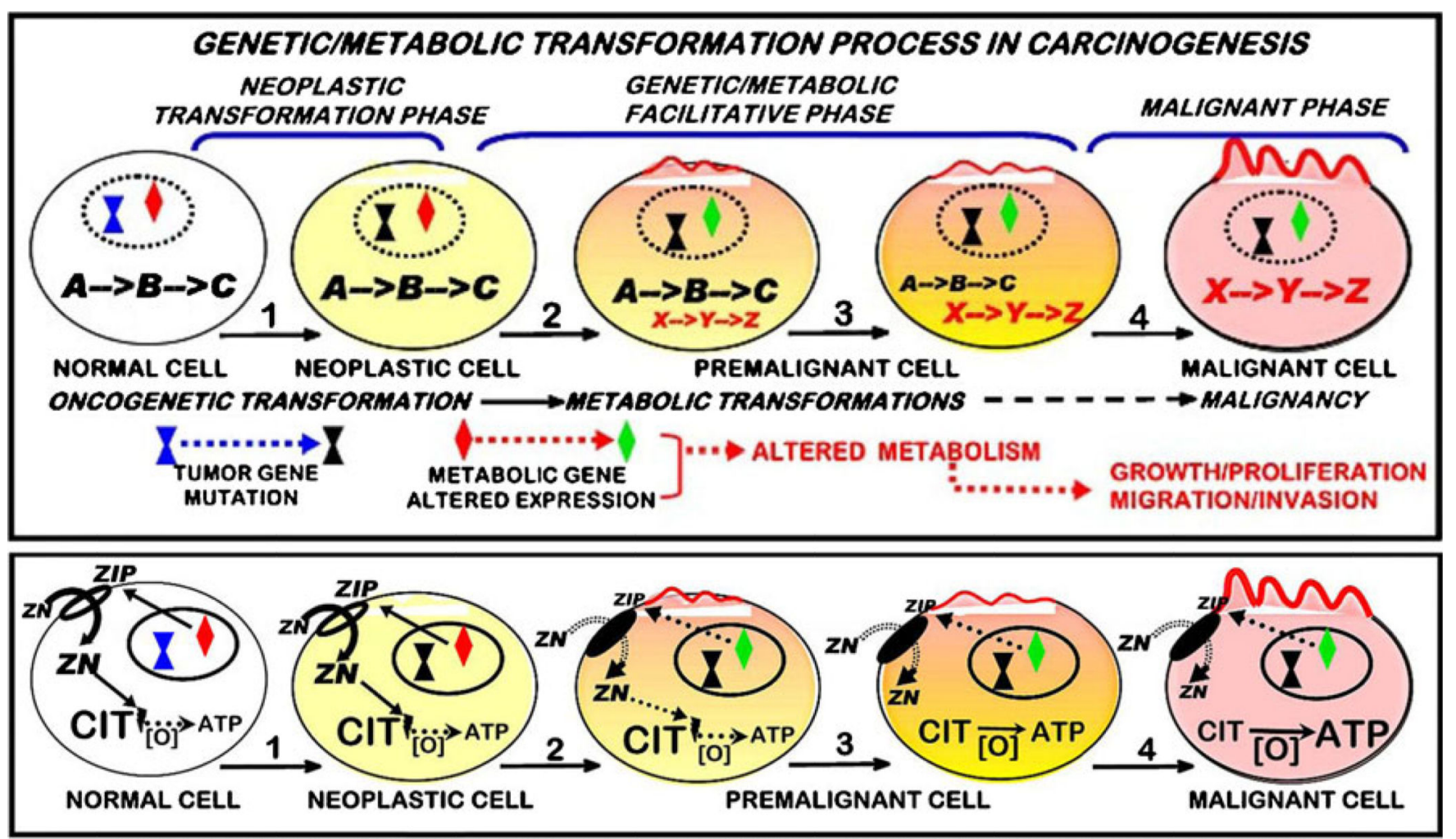

GENETIC/METABOLIC CONCEPT OF PROSTATE CANCER CARCINOGENESIS

Fig. 1.

Genetic/metabolic transformation concept of carcinogenesis and its representation by prostate cancer 